\section{RX (mania) de toráx nas emergências pediátricas}

Ao editor,

Apreciei o artigo de Sarria et al. ${ }^{1}$ sobre o difícil diagnóstico radiológico das pneumopatias agudas infecciosas. Tema criativo sem ser necessariamente original, prescindindo de alta tecnologia, mas com uma metodologia imaginativa, análise inspirada dos resultados e não uma conclusão, mas um convite à reflexão sobre um problema que está à vista de todos nós, todos os dias e todas as horas do dia.

Há muito que faço essas reflexões por escrito e em voz alta, chamando a atenção para a RX mania (de tórax e também de seios da face) que ocorre nos nossos serviços de emergência. Crianças com tosse, febre e/ou chiado no peito raramente escapam do RX. A imagem é previsível e de interpretação dificílima, que não distingue os processos virais dos bacterianos, mas acaba servindo de justificativa para o não emprego de antibióticos ("é só catarro") ou pretexto para a prescrição de antimicrobianos ("tem foquinhos de broncopneumonia"), na grande maioria dos casos feito de maneira abusiva. Essa conduta encarece e retarda o atendimento, obriga a família a permanecer horas à espera do procedimento, atravancando o pronto-socorro e, não raro, atrapalhando o plantonista do horário seguinte, que não foi o que pediu o exame e tem de começar tudo de novo.

Não é só caso de opinião pessoal. A prestigiosa British Thoracic Society, nas suas "guidelines", estabelece que "achados radiológicos são indicadores pobres da etiologia", já que ocorrem tanto nos casos virais como bacterianos ${ }^{2}$. o RX deve ser reservado para casos graves, complicados ou com semiologia sugestiva de consolidação. Para os casos intermediários, de dúvida, melhor recurso é o estudo hematológico e leucograma (leucócitos > 15.000 e neutrófilos > 10.000), o VHS (mais de 30 ) e a Proteína C reativa, não tão facilmente disponível (acima de 9 e especialmente acima de 12). Mas a base de todo o raciocínio continua sendo determinar a intensidade do estado infeccioso (impressão geral) e o acompanhamento pelo pediatra ${ }^{3}$. Só assim se pode melhorar a qualidade do diagnóstico e, conseqüentemente, o uso racional dos antibióticos, como concluem os autores deste artigo original.

\section{Jayme Murahovschi}

Presidente do Departamento de Pediatria Ambulatorial da SBP. Professor de Pediatria da Faculdade de Ciências Médicas de Santos.

\section{Referências}

1. Sarria E, Fischer GB, Lima JAB, Mena Barreto SS, Flôres JAM, Sukiennik R. Concordância no diagnóstico radiológico das infecções respiratórias agudas baixas em crianças. J Pediatr (Rio J). 2003;79:497-503.

2. McIntosh LK. Current concepts: community-acquired pneumonia in children. N Engl J Med. 2002;346:429-37.

3. BTS guidelines for the management of community acquired pneumonia in childhood. Thorax. 2002;57:1-24.

\section{Resposta dos autores}

Agradecemos ao Prof. Murahovschi o interesse no nosso artigo.

Apesar dos grandes avanços científicos da humanidade, particularmente nos últimos 200 anos, no início do século XXI, o diagnóstico de pneumonia em crianças continua sendo um desafio.

É claro que avançou-se de maneira importante na compreensão da fisiopatologia, da epidemiologia e dos fatores de risco, mas nos elementos práticos do diagnóstico e da determinação etiológica, os avanços não acompanharam as necessidades. A perversidade dessa realidade é que os países desenvolvidos, com mais recursos econômicos e disponibilidade de tecnologia, tiveram uma queda expressiva da mortalidade ${ }^{1}$, fundamentalmente pela melhora nas condições socioeconômicas das suas populações. Nos países em desenvolvimento, as determinantes sociais e ambientais condicionam outra realidade, na qual as taxas de mortalidade por pneumonia continuam elevadas.

A estratégia da OMS/OPAS para o controle das infecções respiratórias agudas (IRA), hoje integrada à estratégia AIDPI, permitiu melhorar a captação e o tratamento precoce de casos de pneumonia nos países do mundo em desenvolvimento. A diminuição dos custos diretos em recursos tecnológicos e a maior abrangência se sustentaram no uso de sinais clínicos para o diagnóstico (fundamentalmente tiragem e taquipnéia) e no uso de antibióticos orais ambulatorialmente ${ }^{2}$.

Nessa estratégia, os argumentos para prescindir do radiograma de tórax (RXT) e da realização de exames complementares baseiam-se nos pontos críticos do diagnóstico: 1) a variabilidade interobservadores na interpretação radiológica; 2) a diferenciação entre vírus e bactéria, tanto com os raios $X$ quanto com os exames laboratoriais (hemograma, VSG, proteína $C$ reativa), é questionável.

Com relação aos exames complementares, estudos recentes demonstram que falta compreender melhor os mecanismos inflamatórios que ocorrem nas infecções ${ }^{3}$. As características genéticas do indivíduo e o equílibro entre os 
mediadores pró-inflamatórios e inflamatórios determinarão a resposta inflamatória de fase aguda. Lamentavelmente, nem todos os microorganismos desencadeiam resposta inflamatória de fase aguda, e existe variabilidade entre aqueles que desencadeiam a resposta. Essas explicações, longe de ratificar a pouca utilidade dos exames conhecidos, permitem entender melhor as limitações dos mesmos, o que poderia conduzir futuramente a otimizá-los. Nos guidelines baseados em evidências disponíveis ${ }^{4}$, não se recomenda o uso rotineiro de exames complementares de resposta de fase aguda (nível de evidência I: boas revisões sistemáticas ou estudos rigorosos).

Não se pode negar as limitações do RXT no diagnóstico de pneumonia, mas isso não é razão para satanizar o mesmo. Devemos diferenciar limitações cognitivas de abusos na indicação, assim como erros de interpretação de justificativas infundadas para o uso de antibióticos diante de qualquer imagem nos pulmões. O RXT continua como a ferramenta diagnóstica mais importante no diagnóstico de pneumonia. Dentro da própria OMS, o Grupo de Vacinas faz essa consideração e, atualmente, desenvolve um estudo que pretende padronizar os achados radiológicos no diagnóstico de pneumonia em crianças, para logo utilizar os resultados no estudo que avaliará o impacto da vacina contra o pneumococo na ocorrência da doença 5 .

Nos casos de pneumonia leve, diagnosticados ambulatorialmente e que não precisam de internação, os guidelines recomendam apenas o uso dos sinais clínicos usados pela OMS para o diagnóstico (nível de evidência II: estudos prospectivos), prescindindo do RXT. Igualmente, não recomendam a realização de RXT no seguimento (nível de evidência II). Já na criança com pneumonia grave ou complicada, a realização de RXT deve ser individualizada, dependendo das características e da gravidade de cada caso.

O uso de sinais clínicos na estratégia para o controle das IRA, embora útil, também apresenta limitações. Existe uma variabilidade entre observadores quanto à determinação de sinais clínicos. Segundo uma revisão sistemática publicada no JAMA, os valores de Kappa para sinais clínicos representavam uma concordância entre regular e boa ${ }^{6}$. Esses valores são similares com a concordância interobservadores no uso de RXT no diagnóstico de pneumonia descrita em diversos estudos, incluído o nosso. A sensibilidade e a especificidade dos sinais clínicos, comparados ou associados com RXT, também foram similares em diversos estudos, segundo relatou o grupo ad hoc sobre pneumonia em países em desenvolvimento 7 .

A incapacidade dos sinais clínicos em diferenciar entre etiologia viral ou bacteriana conduz a um uso de antibióticos além do ideal. Em comunicação pessoal (2001), o Dr. Frank Shann, membro do grupo original que propôs o uso dos sinais clínicos, comentava que, cientes disso, naquele momento pesou mais a elevada mortalidade nos países em desenvolvimento no balanço da decisão. O problema é que a taxa de resistência ao co-trimoxasol aumentou expressivamente nos últimos vinte anos, e à amoxacilina vem aumentando rapidamente. Igualmente, o aumento na incidência e prevalência de sibilâncias em países em desenvolvimento faz necessário a revisão das normas, pois tiragem e taquipnéia sempre estão presentes na crise de sibilância. Nesse sentido, o Dr. Yehuda Benguigui, principal funcionário da OPAS, vinculado com o desenvolvimento e a implementação das normas de controle das IRA, também em comunicação pessoal (2001), afirmava que as normas estão em revisão, e ambos os elementos, resistência aos antibióticos e aumento das sibilâncias, serão integrados. Os guidelines consideram que a associação de sibilâncias exclui inicialmente o uso de antibióticos (nível de evidência II).

Dois comentários finais. A educação médica pode desempenhar um papel importante em toda essa situação. É preciso fortalecer as estratégias de ensino da medicina ambulatorial e diminuir o viés de sublimar o ensino no ambiente hospitalar. As vezes, o abuso de médios diagnósticos na prática pretende compensar deficiências no uso de técnicas diagnósticas básicas, pouco estimuladas em ambientes com maior disponibilidade de recursos.

Por último, alguns elementos subjetivos devem ser considerados. A falta de condições para o trabalho médico na maioria das salas de pronto-atendimento dos hospitais públicos pesa no desempenho dos profissionais. A falta de uma rede pública melhor estruturada predispõe ao uso indevido das salas de emergência e à sobrecarga de pacientes nas mesmas. Por um lado, os plantonistas dispõem de pouco tempo para diagnósticos mais precisos e, por outro, aumentam os processos contra médicos. É cada vez mais freqüente o exercício de uma prática médica defensiva, e isso conduz a um uso maior de exames e à prescrição generosa de antibióticos, independentemente das evidências científicas.

\section{Edgar Sarria}

Mestre. Pediatra. Doutorando em Pneumologia, Serviço de Pneumologia Pediátrica, HCSA.

\section{João B. Lima}

Mestre. Pneumologista pediatra, Serviço Pneumologia Pediátrica, HCSA.

\section{Gilberto B. Fischer}

Doutor. Pneumologista pediatra. Chefe do Serviço de Pneumologia Pediátrica, HCSA. Professor titular, FFFCMPA.

\section{Ricardo Sukiennik}

Mestre. Doutorando em Farmacologia. Pediatra, Serviço de Emergências HCSA. Professor substituto, FFFCMPA.

\section{Referências}

1. Dowell S, Kupronis B, Zell E, Shay D. Mortality from pneumonia in children in the United States, 1939 through 1996. N Engl J Med. 2000;342:1399-407.

2. Benguigui Y. Bases técnicas para la prevención, diagnóstico, tratamiento y control de las IRA en el primer nível de atención, em: OPS/OMS: Infecciones Respiratorias Agudas en niños. Serie HCT/AIEPI-1; 1999. p. 333-352.

3. Urban J, Shapira I, Branski D, Berliner S. Acute phase response in the diagnosis of bacterial infections in children. (Concise Reviews of Pediatric Infectious Diseases) Ped Inf Dis J. 2004; 2:159-60.

4. British Thoracic Society. BTS guidelines for the management of community acquired pneumonia in childhood. Thorax. 2002;57 Suppl 1:1-24.

5. WHO Pneumonia Vaccine Trial Investigator's Group. Standardization of interpretation of chest radiographs for the diagnosis of pneumonia in children, WHO/V\&B/01.35; 2001.

6. Margolis P, Gadomski A. Does this infant have pneumonia? The Rational Clinical Examination. JAMA. 1998;279:308-13.

7. Pechère JC. Community Acquired Pneumonia in Children. International Forum Series. United Kingdom: C.M.P. Publication; 1995. 


\section{Osteoporose é uma doença que acomete crianças e adolescentes?}

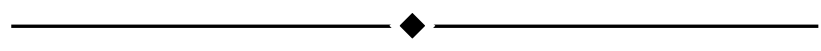

Prezado Editor:

Lemos com grande interesse o artigo de Campos et al. relativo a osteoporose na infância e adolescência, recentemente publicado no Jornal de Pediatria ${ }^{1}$. No artigo em foco, foi efetuada uma excelente revisão da literatura, contemplando com abrangência o assunto de forma objetiva e esclarecedora. Entretanto, observa-se um pequeno viés na descrição dos métodos de imagem, particularmente relacionado com a DEXA (emissão dupla de raios- $X$ ), onde os autores indicaram que a medida de densidade óssea (DMO) é expressa em $\mathrm{g} / \mathrm{cm}^{3}$. A DMO avaliada por este método é expressa em $\mathrm{g} / \mathrm{cm}^{2}$, sendo fruto da razão entre o conteúdo mineral ósseo (CMO) em gramas e a área total ( $\mathrm{A}$ ) da região investigada, expressa em $\mathrm{cm}^{2}$.

Outro aspecto ressaltado pelos autores e de grande interesse foi o enfoque à orientação dietética, perfeitamente estruturada e confirmada por outros relatos, inclusive por trabalho nosso a ser publicado brevemente (Silva et al., 2004) ${ }^{2}$. Já no tópico "Atividades físicas", ressaltamos que o termo "fisioterapia com pesos" seria melhor expresso por "exercícios físicos com peso" (musculação), anteriormente já retratados em revisão da literatura 3,4 , relacionando esporte e suas implicações na saúde óssea de atletas adolescentes 5 .

No que se refere aos períodos críticos de aquisição do capital mineral ósseo, Campos et al. ${ }^{1}$ relatam intensa mineralização durante os primeiros anos da vida e na adolescência (entre 11 e 14 anos nas meninas e 13 e 17 anos nos meninos). $O$ profundo interesse na área nos conduziu a uma investigação com adolescentes do sexo masculino, e os resultados indicaram intenso anabolismo ósseo entre as faixas etárias de 14 a 16 anos e quando os mesmos atingiam o estágio de maturação sexual G4.

Foi com grande satisfação que redigimos esta carta endereçada aos autores do referido artigo, o qual nos proporcionou esclarecimentos e a oportunidade de conjugar conhecimentos que acreditamos serem imprescindíveis para a evolução do entendimento sobre o metabolismo ósseo na infância e adolescência, pois embora as manifestações clínicas terminais relacionadas aos quadros de osteoporose ocorram principalmente na terceira idade, sua predisposição tem início na infância e adolescência, períodos de máxima incorporação do capital mineral ósseo.

Tamara B. L. Goldberg

Doutora. Professora assistente, Departamento de Pediatria, Faculdade de Medicina Botucatu - UNESP.

\section{Carla C. Silva}

Mestranda em Pediatria pelo Programa de Pós-Graduação em Pediatria, Faculdade de Medicina Botucatu - UNESP. Bolsista CNPq.

\section{Referências}

1. Campos LMA, Liphaus BL, Silva CAA, Pereira RMR. Osteoporose na infância e adolescência. J Pediatr (Rio J). 2003;79:481-8.
2. Silva CC, Teixeira AS, Goldberg TBL. Impacto da ingestão de cálcio sobre a mineralização óssea em adolescentes. Rev Nutr. No prelo 2004.

3. American Academy of Pediatrics, Committee on Sports Medicine and Fitness. Strength training by children and adolescents. Pediatrics. 2001;107:1470-2.

4. Faigenbaum $A D$. Strength training for children and adolescents. Clin Sports Med. 2000;19:593-619.

5. Silva CC, Teixeira AS, Goldberg TBL. O esporte e suas implicações na saúde óssea de atletas adolescentes. Rev Bras Med Esporte. 2003;9:426-38.

\section{Resposta dos autores}

Prezado Editor,

Agradecemos as considerações de Goldberg \& Silva referentes à nossa revisão "Osteoporose na infância e Adolescência"1 e esclarecemos algumas questões referentes ao mesmo. Como foi descrito no nosso artigo e comentado por dois leitores, a massa óssea é avaliada em termos de conteúdo mineral ósseo (CMO, em gramas) e densidade mineral (DMO, resultante da relação do $\mathrm{CMO}$ em gramas e área total em $\left.\mathrm{cm}^{2}\right)$. Mesmo quando a DMO $\left(\mathrm{g} / \mathrm{cm}^{2}\right)$ é ajustada pela área escaneada, isto não corrige as diferenças no espessamento ósseo. Deste modo, principalmente em crianças, a verdadeira densidade mineral óssea é superestimada em ossos grandes e subestimada em ossos pequenos. Tentando evitar este problema, alguns autores desenvolveram modelos matemáticos para estimar o volume ósseo $\left(\mathrm{cm}^{3}\right)$ em vez da área óssea $\left(\mathrm{cm}^{2}\right)^{2-5}$. Este método de análise reduz os efeitos de confusão do tamanho do osso. Carter et al. ${ }^{5}$ denominam o mesmo como "densidade mineral óssea aparente" (BMAD, $\mathrm{g} / \mathrm{cm}^{3}$ ). Apesar desta metodologia aparentemente ser mais correta, porém, mais difícil para ser realizada, na prática clínica, e mesmo nas nossas pesquisas clínicas em crianças adolescentes normais ${ }^{6,7}$ e com patologias crônicas ${ }^{8}$, acabamos utilizando o resultado proveniente do densitômetro, cuja densidade mineral óssea é resultante do $\mathrm{CMO}$ e área do local analisado $\left(\mathrm{cm}^{2}\right)$.

A atividade física, em especial os exercícios contra a gravidade, deve ser uma das principais recomendações para prevenção e tratamento da osteoporose, principalmente na faixa etária pediátrica, época em que o pico de massa óssea é atingido. Os autores do artigo de revisão em questão concordam com a sugestão da utilização do termo "exercícios físicos com peso" (musculação) em vez de "fisioterapia com peso". A musculação é uma importante estimuladora do aumento da massa óssea, porém, algumas vezes a sua utilização é limitada pela baixa idade e estadiamento puberal, devendo ser sempre realizada com supervisão profissional. Embora existam controvérsias, outras atividades físicas sem impacto podem também estimular a massa óssea. Lima et al. ${ }^{7}$, em um estudo realizado na disciplina de Reumatologia da FMUSP, estudaram o metabolismo ósseo e a composição corporal em três grupos de adolescentes do sexo masculino: atletas praticantes de atividade de impacto, de atividade sem impacto e controles normais. A maior DMO foi demonstrada no grupo praticante de exercícios com impacto, e mesmo os adolescentes praticantes de atividade sem impacto apresentaram DMO superior ao grupo controle. 


\section{Referências}

1. Campos LM, Liphaus BL, Silva CA, Pereira RM. Osteoporose na infância e adolescência. J Pediatr (Rio J). 2003;79:481-8.

2. Kroger $\mathrm{H}$, Kotaniemi A, Vainio $P$, Alhava $E$. Bone densitometry of the spine and femur in children by dual-energy $x$-ray absorptiometry. Bone Miner. 1992;17:75-85.

3. Bachrach LK. Osteoporosis in childhood and adolescence. In: Marcus R, Feldman D, Klesey J, editors. Osteoporosis. 2nd ed. San Diego:Academic Press; 2001. p.151-67.

4. Lu PW, Cowell CT, Loyd-Jones SA, Briody JN, Howman-Giles R. Volumetric bone mineral density in normal subjects, aged 5-27 years. J Clin Endocrinol Metab. 1996;81:1586-90.

5. Carter DR, Bouxsein ML, Marcus R. New approaches for interpreting projected bone densitometry data. J Bone Miner Res. $1992 ; 7: 137-45$.

6. Pinto ALS, Takayama L, Carazzato JC, Pereira RMR, Lima FR. Bone density and body composition in adolescent female swimmers. Arthritis Rheum. 2000;43:S393.

7. Lima F, De Falco V, Baima J, Carazzato JG, Pereira RM. Effect of impact load and active load on bone metabolism and body composition of adolescent athletes. Med Sci Sports Exerc. 2001;33:1318-23.
8. Pereira RM, Corrente JE, Chahade WH, Yoshinari N. Evaluation by dual X-ray absorptiometry (DXA) of bone mineral density in children with juvenile chronic arthritis. Clin Exp Rheumatol. 1998; 16:495-501.

\section{Lúcia M. A. Campos}

Mestre. Médica assistente, Unidade de Reumatologia, Instituto da Criança, Hospital das Clínicas, FMUSP.

\section{Bernadete L. Liphaus}

Mestre. Médica assistente, Unidade de Reumatologia, Instituto da Criança, Hospital das Clínicas, FMUSP.

Clovis A. A. Silva

Doutor. Médico Chefe da Unidade de Reumatologia, Instituto da Criança, Hospital das Clínicas, FMUSP.

\section{Rosa M. R. Pereira}

Doutora. Professora assistente, FMUSP. Chefe do Laboratório de Doenças Osteometabólicas, Departamento de Reumatologia, FMUSP. 